\title{
Invasive aspergillosis of the central nervous system in immunocompetent patients in Saudi Arabia: Case series and review of the literature
}

\author{
Mahmoud S. Taha, MD, Mabmoud I. Haddad, MD, Ali A. Almomen, MD, Marwa M Abdulkader, MD, Rami A. Alhazmi, \\ $M D$.
}

\begin{abstract}

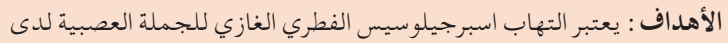

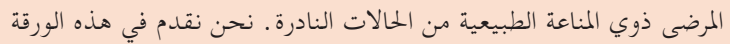

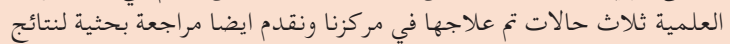

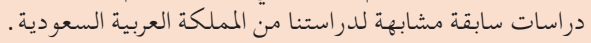

المنهجية: قمنا في هذه الدراسة بمراجعة رجعية لكل حالات التهاب التهبي

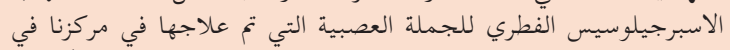

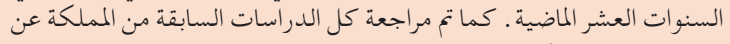

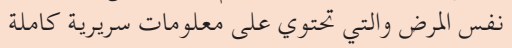

النتائج: تم علاج ثلاث حالات من التهاب الجملة العصبية الفطري

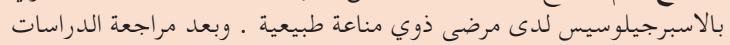

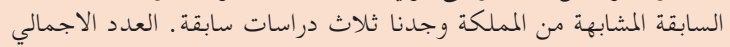

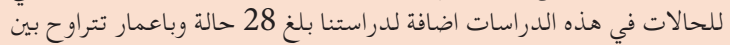

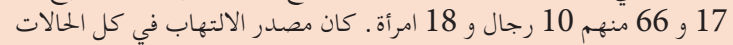

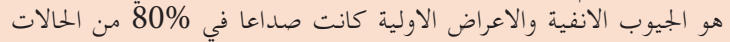

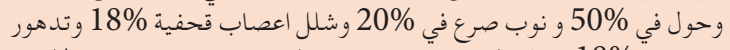

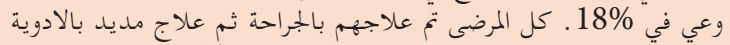

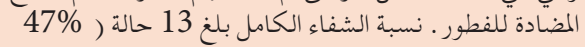

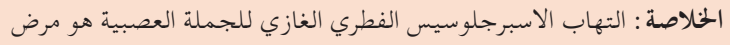

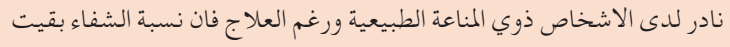

Objective: Invasive aspergillosis of the central nervous system in immunocompetent patients is a rare disease. We present in this study three cases that were treated in our centre and reviewed the results of similar studies from Saudi Arabia.

Methods: We retrospectively reviewed all cases of invasive aspergillosis of the central nervous system (CNS) that were treated in our hospital in the last 10 years. We also reviewed the literature for any similar series published from Saudi Arabia.

Results: We had three cases treated in our centre and we also found three similar case series in the literature. Total number of cases, including our series was 28 , age range from 17 to 66,10 men and 18 women.
The source of infection was nasal sinuses in all cases. Initial presentations were variable and included headache ( $80 \%$ of cases), proptosis or diplopia $(50 \%$ of cases), seizures ( $20 \%$ of cases), cranial nerve palsies (18\% of cases) and acute deterioration in level of consciousness (18\% of cases). All patients underwent surgery followed by long course of antifungal treatment. Clinical outcome was reported as cured or no recurrence in 13 cases $(47 \%)$.

Conclusions: Invasive aspergillosis of CNS is a rare disease in immunocompetent patients. Despite treatment prognosis remains unfavourable in many cases.

Neurosciences 2021; Vol. 26 (4):379-384 doi: 10.17712/nsj.2021.4.20210081

From the Department of Neurosurgery (Taha, Haddad), Department of Head and Neck Surgery (Almomen), Department of Pathology (Abdulkader), Department of Medical Imaging (Alhazmi), King Fahad Specialist Hospital, Dammam, Kingdom of Saudi Arabia

Received 7th June 2021. Accepted 22nd August 2021.

Address correspondence and reprint request to: Dr. Mahmoud S. Taha, Department of Neurosurgery, King Fahad Specialist Hospital, Dammam, Saudi Arabia,E-mail:mtaha66@hotmail.com ORICID ID: orcid.org/0000-0002-3578-9393

Tnvasive aspergillosis (IA) is the most common invasive mould infection, particularly among immunocompromised patients. Aspergillus fumigatus accounts for almost $90 \%$ of infections in humans. However, other Aspergillus species, such as A. flavus, A. terreus and $A$. niger, were more commonly isolated in cases involving immunocompetent patients. ${ }^{1}$ Cases of

Disclosure. The authors declare no conflicting interests, support or funding from any drug company. 
of IA of the central nervous system (CNS) infections in immunocompetent patients are rare and mainly reported in tropical and subtropical geographic areas, including Southeast Asia and the Middle East. ${ }^{2}$

The most common primary infection site of IA of CNS in immunocompetent patients is usually the paranasal sinuses (sinus aspergillosis). From there, the infection can invade the skull base and orbit and then locally infiltrate the intracranial cavity. This invasion can lead to different pathological changes in the CNS, including granuloma or abscess formation, meningeal inflammation and cerebrovascular insults (ischaemic stroke, or intracranial haemorrhage due to vascular thrombosis, or mycotic aneurysm). ${ }^{3,4}$

Clinical presentations of IA of CNS can be indolent or acute with variable symptoms, depending on the location of the infection in the CNS and the ensuing pathological changes. ${ }^{2}$ The diagnosis of IA of CNS is usually difficult, since clinical presentations can vary, and radiological findings may mimic invasive skull base lesions, high-grade brain tumours, and other causes of CNS infections or abscess. ${ }^{2-4}$ Sampling of the infected area (sinuses or intracranial extension) for bacterial, mycobacterium, fungal and histopathology are essential for diagnosis. ${ }^{2}$ When diagnosis of IA of CNS is confirmed, surgical excisions of infected tissue of the paranasal sinuses, skull base bone and brain abscesses/granuloma should be performed when feasible. ${ }^{4}$ Following surgery, an extended course of antifungal therapy should be prescribed. However, initiation of empirical antifungal therapy on suspicion is recommended due to high mortality. ${ }^{1}$ Prognosis remains poor in a the majority of IA of CNS cases due to difficulty in achieving a complete resection due to the location of the brain invasion and involvement of intracranial vessels. In addition, some antifungal medications have limited ability in cerebrospinal fluid penetration. ${ }^{2,4}$

In this paper, we present a case series of IA of CNS in immunocompetent patients from our centre. We have also searched the literature for similar studies of the disease in Saudi Arabia in the last four decades. The overall aim was to review the clinical presentations, radiological findings, pathological features, mycology studies, treatment and clinical outcomes of IA of CNS in Saudi Arabia.

Methods. We retrospectively reviewed cases of IA of CNS in immunocompetent patients that were treated at our center between 2010 and 2020. We only included cases with confirmed Aspergillosis infections by pathology or cultures of the central nervous system, and are immunocompetent. Cases with HIV infection, malignancy, on chemotherapy or other immunosuppression medications were excluded. Literature search for Aspergillosis infections of the CNS in Saudi Arabia were done by using PubMed. Only case series of IA of CNS in immunocompetent patients, and had full clinical details were included in our review. Cases from our series and from the historical case series studies were collected and analysed for age, gender, clinical presentations, radiological findings, pathology reports, mycology results, surgical approaches, medical treatment, and clinical outcome. Categorical data were presented as frequency and percentage.

Results. We had 3 cases in total from our centre; all were males in their thirties $(30,33$ and 36
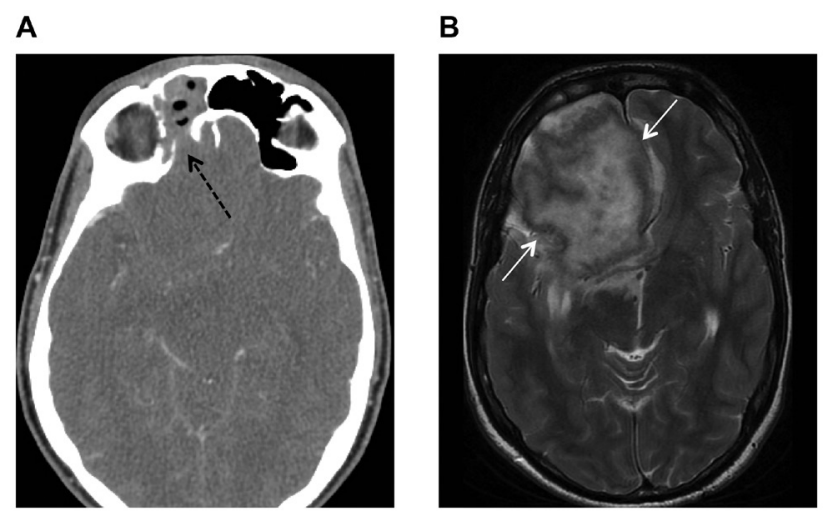

C

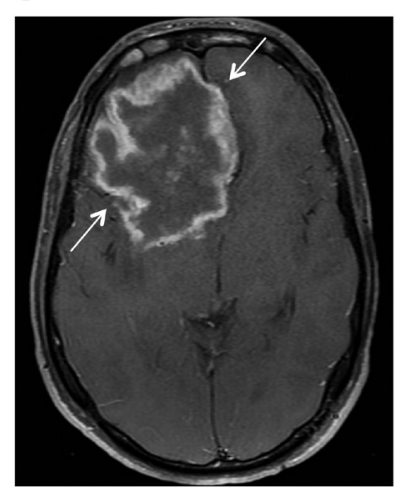

D

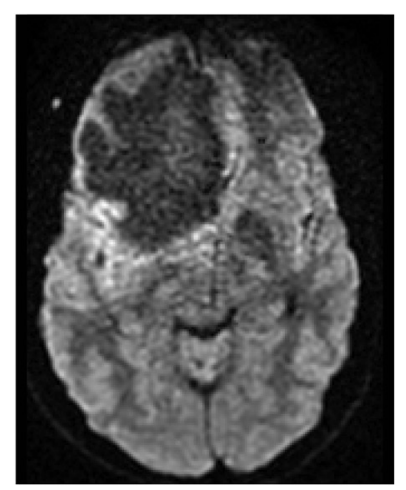

Figure 1 - Axial enhanced brain computed tomography (CT) A) Axial enhanced brain computed tomography (CT) shows hyperdense opacification of the right frontal paranasal sinus with erosion of its posterior wall (black dashed arrow), B) Brain magnetic resonance imaging MRI scan Axial T2-weighted and, C) MRI T1-weighted post contrast show a large intra-axial lesion in the right frontal lobe with irregular thick peripheral enhancement (white arrows), D) MRI diffusion-weighted imaging shows no corresponding central diffusion restriction. 
Table 1 - Summary of studies on IA of CNS in immunocompetent patients in Saudi Arabia including the current study.

\begin{tabular}{|c|c|c|c|c|c|c|c|}
\hline Study period & $\mathrm{n}$ & $\begin{array}{c}\text { Age: } \\
\text { mean(range) }\end{array}$ & $\begin{array}{c}\text { Male/ } \\
\text { female }\end{array}$ & Aspergillus spp. & Treatment anti-fungal & Clinical outcome & Follow up (months) \\
\hline $\begin{array}{l}\text { Mohan et al }{ }^{7} \\
(1987-92)\end{array}$ & 4 & $42.2(30-60)$ & $2 / 2$ & A. flavus (4) & Surgery/Amphotericin B & $\begin{array}{l}3 \text { no recurrence } \\
1 \text { death }\end{array}$ & 12-24 months \\
\hline $\begin{array}{l}\text { Ur-Rahman } \\
\text { et al. }(1983-94)\end{array}$ & 9 & $47.3(26-66)$ & $1 / 8$ & $\begin{array}{l}\text { A. flavus (6) } \\
\text { A. fumigatus (3) }\end{array}$ & $\begin{array}{l}\text { Surgery/Amphotericin B plus } \\
\text { flucytosine then itraconazole }\end{array}$ & $\begin{array}{c}1 \text { cured } \\
6 \text { residual or lost } \\
\text { f/up } \\
2 \text { death }\end{array}$ & 1-36 months \\
\hline $\begin{array}{l}\text { Baeesa et al }{ }^{9} \\
(2000-12)\end{array}$ & 12 & $32(17-50)$ & $4 / 8$ & not defined & $\begin{array}{l}\text { Surgery/Amphotericin B plus } \\
\text { itraconazole or variconazole }\end{array}$ & $\begin{array}{l}8 \text { cured } \\
2 \text { residual or } \\
\text { recurrence } \\
2 \text { deaths }\end{array}$ & $12-50$ months \\
\hline $\begin{array}{l}\text { Taha et al }(2012- \\
18)\end{array}$ & 3 & $33(30-36)$ & $3 / 0$ & not defined & $\begin{array}{l}\text { Surgery/Amphotericin B plus } \\
\text { variconazole }\end{array}$ & $\begin{array}{l}1 \text { cured } \\
2 \text { deaths }\end{array}$ & $1-18$ months \\
\hline
\end{tabular}

A

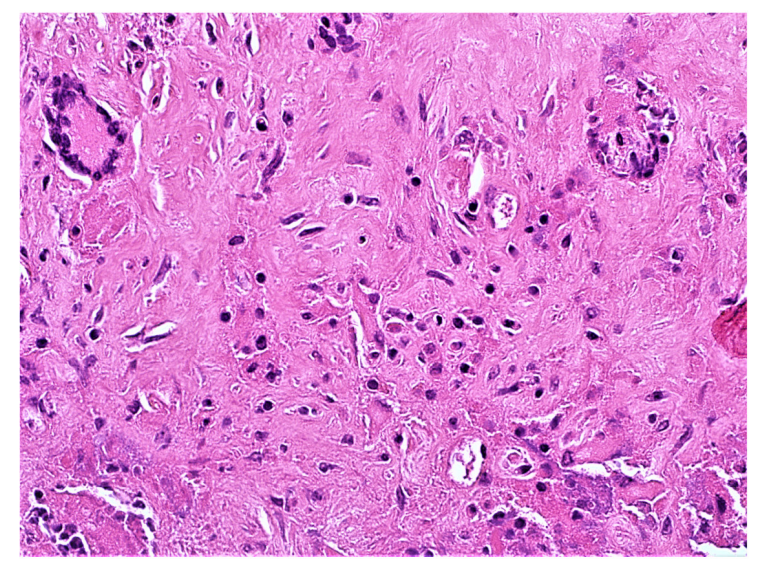

\section{B}

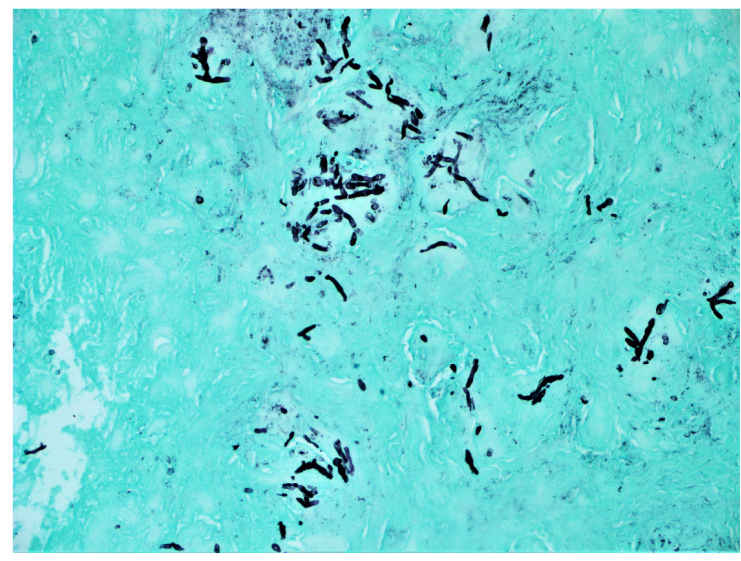

Figure 2 - Hematoxylin- and eosin-stained sections (magnification, 40x) showing A) neuroglial tissue containing ill-defined granulomata with multinucleated giant cells. Thin hyphae with regular septation and branching at a $45^{\circ}$ angle are noted, B) Grocott's methenamine silver stain demonstrating thin hyphae and spores (magnification, 20x).

years) and hailed from the Eastern region of Saudi Arabia. All cases were immunocompetent with no history of malignancies, recurrent infections or immunosuppression medications. All tested negative for HIV infection. Two patients had previous history of Aspergillus sinusitis that was treated with surgery and antifungal therapy. The 3 cases all presented with a short history (2-4 weeks) of headache and vomiting, with one reporting of seizure. Radiologically, 2 cases presented with brain abscess and one with skull base lesions. All were treated with aggressive surgery and antifungal therapy after confirming the diagnosis with pathology and cultures. The 2 cases of brain abscess features died within a few months due to increased intracranial pressure and strokes as a result of intracranial vascular thrombosis. The third case survived with excellent outcomes following a long course (12 months) of antifungal treatment.
From the literature, we found three similar studies of IA of CNS in immunocompetent patients from Saudi Arabia that were published between 1980 and 2020 (Table 1). ${ }^{7-9}$ The total combined number of cases of these studies, including ours, was 28 . Patients had an age range of 17 to 66 and included 10 males and 18 females. Seven cases (25\%) had previous history of Aspergillus sinusitis, and $90 \%$ had nasal symptoms for months or years prior to CNS infection. The initial presentations were headache in $80 \%$ of cases, proptosis or diplopia (due to orbital invasion) in $50 \%$, seizures in $20 \%$, cranial nerve palsies (mainly anosmia or decrease vision) in $18 \%$ and acute deterioration in consciousness level (due to brain haemorrhage) in 18\%. All cases underwent CT with contrast, MRI or both for diagnosis. Evidence of paranasal sinusitis (multiple or isolated) was observed in all cases. Orbital invasions were seen in half of the 


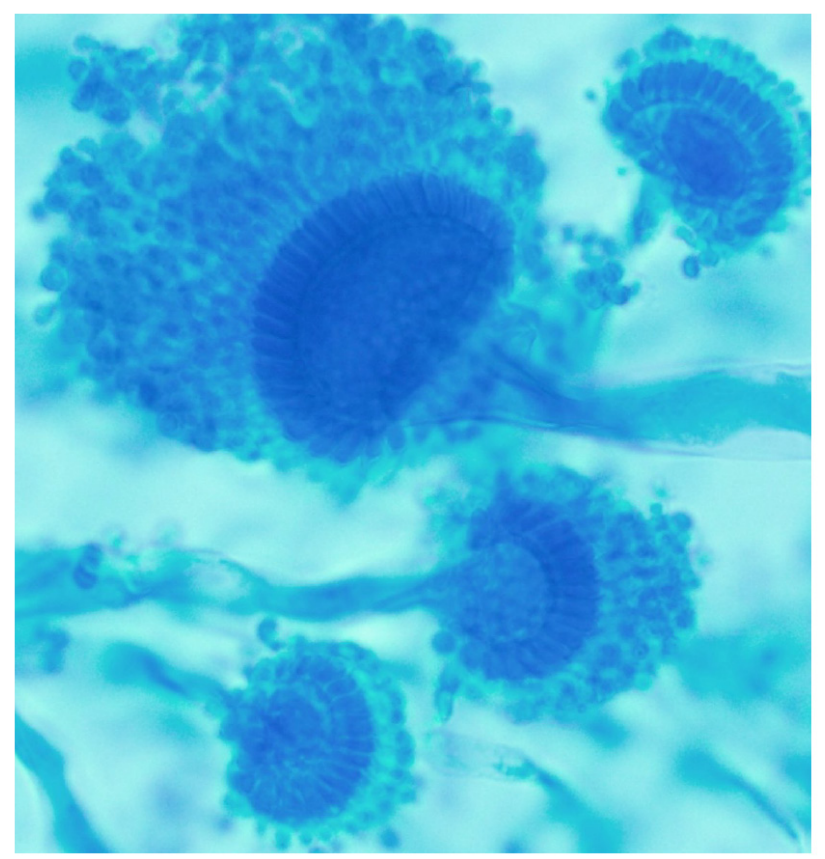

Figure 3 - Detection methods for mycology: Specimens were inoculated on both selective fungal media (Mycosel, Becton, Dickenson and Company, MD, USA) and general fungal media Sabouraud Dextrose Agar (Titan Biotech Ltd, India) and incubated at $28^{\circ} \mathrm{C}-30^{\circ} \mathrm{C}$ for up to 4 weeks. Cultures with fungal growth were further worked up for mould identification using tease mount, scotch tape preparation and potato dextrose agar media for conidiation. The culture grew rapidly, producing a greengrey colony. The mould produced septate hyaline hyphae with smooth conidiophores supporting a dome-shaped vesicle. A row of phialides on the upper surface of the vesicle bore chains of round conidia in a columnar fashion characteristic of Aspergillus fumigatus.

cases. Intracranial invasions were seen in all cases with evidence of skull base erosions. Intracranial invasions were seen in the anterior fossa in $78 \%$ of cases and in the sellar/parasellar regions in $26 \%$ of cases. Radiologic images revealed peripheral enhancement in $20 \%$ of cases (case 1 in our series) and homogenous enhancement in $80 \%$ of cases (case 2 in our series). Patients with acute presentation due to cerebrovascular insults (4 cases) had MR angiography or catheter angiography. Two had intracerebral haemorrhage with no apparent vascular lesions, and 2 had subarachnoid haemorrhage due to mycotic aneurysms. ${ }^{7-9}$ All patients in these studies underwent surgery for debridement and attempted resection of all infected tissue in the paranasal sinuses, skull base and brain. The open approach for the sinuses and skull base was used in previous series, ${ }^{7,8}$ whereas a combined endoscopic transnasal and transcranial/skull base approaches were used in the last 2 series. Partial
A

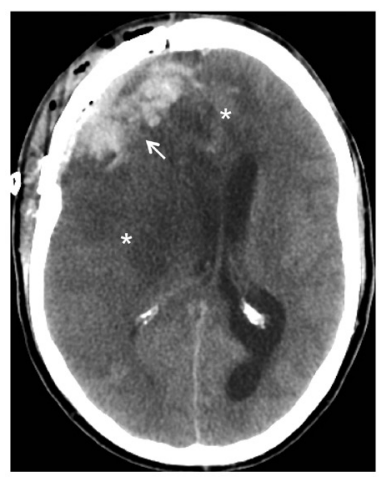

B

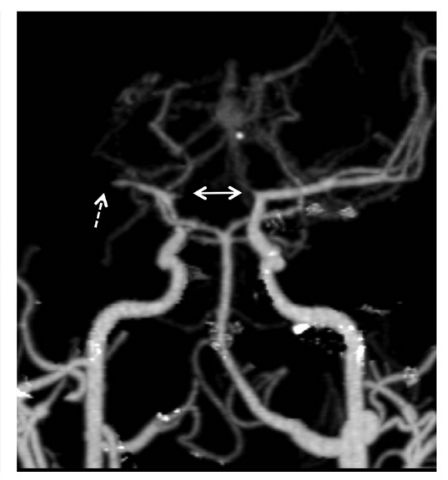

Figure 4 - Axial plain brain CT shows (A) post-craniotomy changes in the right frontal aspect of the skull with haemorrhagic collection within the surgical bed (white arrow). Extensive ischemic lowattenuation tissue swelling in the right cerebral hemisphere and left frontal lobe is also observed (asterisks) along with a left-sided midline shift. (B) 3-dimensional reformatted MIP image from CTA shows occlusion of the right M1 segment (dashed arrow) and bilateral ACA (bidirectional arrow).

resection of the intracranial lesions due to invasion of cavernous sinuses or vessels was achieved in most cases. Diagnosis of IA in all the series was based on pathology and cultures (Figure $2 \& 3$ ). The antifungal therapies prescribed are shown in Table 1. Therapy usually started with amphotericin B with or without another antifungal for 1-3 months, followed by oral itraconazole or voriconazole for 6-18 months. With regards to outcome, death was reported in 7 patients $(25 \%)$ due to progression of intracranial disease or vascular ischaemia, recurrence, residual or lost follow-up in 8 patients (28\%) and no recurrence in 13 cases (47\%) (Table 1). Here, we present 2 of our 3 cases for illustration purposes.

Reports of 2 demonstrative cases. Case 1. The patient was a 36-year-old male with no significant previous medical history. He was admitted to our hospital after a one-month history of headache, dizziness and vomiting. The patient was fully awake with no neurological deficits. The CT of the head with contrast showed a large right frontal intra-axial lesion with peripheral enhancement and mass effect with evidence of mucosal thickening in the right frontal and ethmoid sinuses (Figure 1A). Brain MRI with contrast showed similar findings, raising suspicion for paranasal sinusitis with intracranial abscess (Figure 1B-1D).

The patient underwent a surgical procedure performed jointly by the neurosurgical and ENT teams. Right frontal craniotomy and partial removal of the infected brain tissue were performed together 


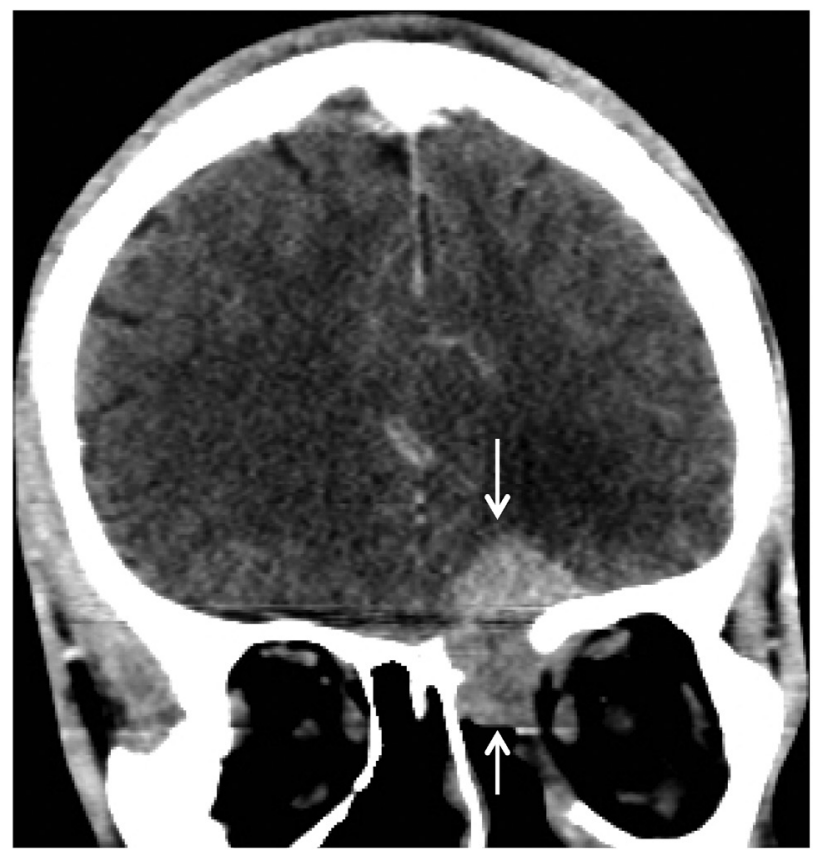

Figure 5 - Enhanced coronal brain CT shows a growing mass centred in the anterior skull base with bone erosions (arrows) along with intracranial, extra-axial and ethmoid sinus soft tissue components.

with transnasal endoscopic surgery and drainage/ debridement of the paranasal sinuses. Specimens from both surgical sites were sent for pathology and cultures. The patient remained clinically stable after surgery. Intravenous antifungal therapy (amphotericin B and voriconazole) was immediately started postoperatively together with a high dose of steroids (dexamethasone $4 \mathrm{mg}$ every $6 \mathrm{~h}$ ) following the confirmation of IA on pathology (Figure 2A and 2B). Tissue cultures also confirmed the diagnosis of aspergillosis (Figure 3).

One week following surgery, the patient deteriorated clinically and became unresponsive. Brain scans showed multiple areas of brain ischaemia, evidence of bilateral cerebral artery thrombosis and brain swelling (Figure 4A and 4B).

The patient underwent right decompression craniotomy and was kept intubated and ventilated in the intensive care unit for maximum medical therapy. The patient continued to deteriorate despite intensive medical therapy and died two weeks following his vascular event.

Case 2. This case involved a 33-year-old male with a previous medical history of fungal sinus infection, which was treated in another hospital 8 years prior. The patient presented with seizures for few weeks. On admission, he

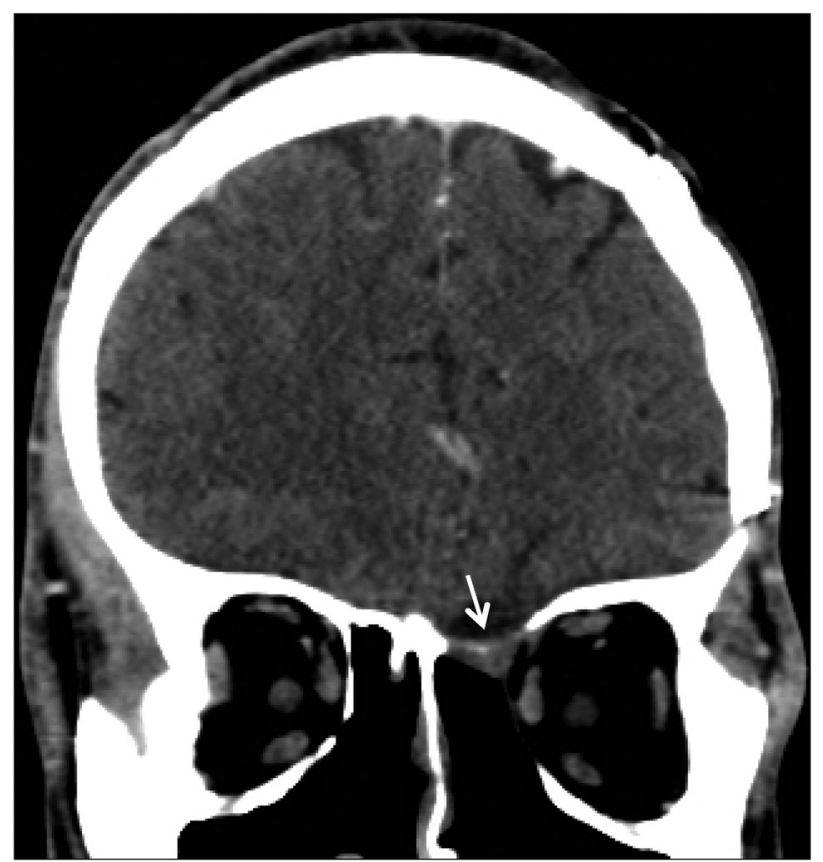

Figure 6 - Enhanced coronal brain CT on post-operative follow up does not show any evidence of recurrence.

was fully awake and had no neurological deficits. The CT of the brain showed a skull base lesion in the left anterior fossa with bony destruction and extension to the left ethmoid and frontal sinus (Figure 5).

The patient underwent urgent combined transcranial left craniotomy and transnasal endoscopic debridement and drainage of the frontal and ethmoidal sinuses. The lesion was completely resected and sent for pathology and cultures. The results confirmed IA in the skull base lesion and paranasal sinuses. The patient did well after surgery and was started on amphotericin liposomal IV and initially on voriconazole IV before transitioning to voriconazole orally at an appropriate dose for 4 weeks and then for a further 12 months in addition to antiepileptic medication (12 months) and dexamethasone (6 weeks). He adhered to follow-up at the outpatient clinic for 18 months and was found clinically stable and symptom-free. His final follow-up images showed no evidence of recurrence of his primary fungal infection (Figure 6).

Discussion. The IA of the CNS is a rare disease that is usually spread from the paranasal sinuses. Sinus aspergillosis has been classified into 4 main types: 
allergic aspergillosis of the sinuses, aspergilloma of the sinus, invasive chronic sinusitis and invasive fulminant sinusitis. The allergic type and sinus aspergilloma do not usually invade the brain. ${ }^{5}$

Almost one-third of immunocompetent patients with chronic IA of the paranasal sinuses have intracranial invasion according to Alrajhi et $\mathrm{al}^{6}$ who investigated cases of chronic IA of paranasal sinuses in Saudi Arabia.

The clinical outcomes of IA of CNS in immunocompetent cases in Saudi Arabia series were similar to a reasonably large series in Pakistan ${ }^{3}$ that included 25 cases between 1991 and 2003. The overall mortality in this study was $28 \%$. The age range was wider (14-74) with a mean age of 36.5 years. The cases were predominantly male in this study (23:2 compared with 10:18 in the Saudi studies). Siddiqui et al (2004) categorised the patterns of cranio-cerebral invasion of IA to 3 types that correlated with outcomes: Type 1, intradural IA that had a $66 \%$ mortality rate; Type 2, extradural intracranial IA with intermediate response and outcome; and Type 3, invasion limited to the orbital wall and cranial base that presented excellent functional outcomes with treatment.

Limitation of our study include small sample size which is expected considering the rarity of the disease. In addition, it is worth mentioning that the treatment methods in the 4 case series were not similar or standard. Examples include open surgery versus endoscopic surgery, and differences in anti-fungal medications and its durations. These differences might have effect on the clinical outcome.

In conclusion, IA of CNS in immunocompetent patients is a disease infrequently encountered in tropical and subtropical areas, including the Middle East. Diagnosis can be difficult and late due to similarity of clinical presentations and diagnostic imaging with other causes of CNS infections and tumours. Despite aggressive surgery and the use of appropriate combined antifungal therapy, the prognosis remains poor, with high mortality and recurrence.

Acknowledgment. The authors would like to thank Ms. Margot Windisch for English language editing.

\section{References}

1. Patterson TF, Thompson, GR, Denning DW, Fishman JA, Hadley S, Herbrecht R, et al. 2016. Practice guidelines for the diagnosis and management of aspergillosis: 2016 Update by the Infectious Diseases Society of America. Clin Infect Dis 2016; 63: e1-e60.

2. Miceli, MH. Central nervous system infections due to aspergillus and other hyaline molds. J Fungi (Basel) 2019; 5: 79.

3. Siddiqui AA, Shah AA, Bashir SH. Craniocerebral aspergillosis of sinonasal origin in immunocompetent patients: clinical spectrum and outcome in 25 cases. Neurosurgery 2004; 55: 602-11; discussion 611-613.

4. Nadkarni T, Goel A. Aspergilloma of the brain: an overview. $J$ Postgrad Med 2005; 51: S37-S41.

5. Al-Bhlal LA. Fungal infection of the nasal cavity and paranasal sinuses: Review of 26 cases. Ann Saudi Med 1996; 16: 615-621.

6. Alrajhi AA, Enani M, Mahasin Z, Al-Omran K. Chronic invasive aspergillosis of the paranasal sinuses in immunocompetent hosts from Saudi Arabia. Am J Trop Med Hyg 2001; 65: 83-86.

7. Kameswaran M, Al-Wadie A, Khurana P, Okafor BC. Rhinocerebral aspergillosis. J Laryngol Otol 1992; 106: 981-985.

8. Ur-Rahmanl N, Jamjoom A, Al-Hedaithy SA, Jamjoom ZA, AlSohaibani MO, Aziz SA. Cranial and intracranial aspergillosis of sino-nasal origin. Acta Neurochir (Wien) 1996; 138: 944-950.

9. Baeesa SS, Bokhari RF, Alghamdi, KB, Alem HB, Al-Maghrabi JA, Madani TA. Invasive aspergillus sinusitis with orbitocranial Extension. Asian J Neurosurg 2017; 12: 172-179. 